\title{
A technique for quantifying and analyzing evoked responses*
}

\section{R. A. ROEMER, T. J. TEYLER, and R. F. THOMPSON}

Department of Psychobiology, University of California, Irvine, California 92664

Two computer programs are discussed. One is used in the analog-to-digital conversion of and averaging of the responses; the other employs full-wave rectification and numerical integration as a method of comparing waveforms.

The computer-supported data collection and analysis methods described here are particularly applicable to the study of grossly recorded activity in neural tracts. These procedures permit an assessment of the overall neural activity which comprises the wave form. A PDP-12 computer is currently being employed to implement these methods. The procedure consists of two separate programs: DATACOL, for on-line data collection, and RUNANAL, for off-line analysis.

Off-line analysis permits many different approaches to the assessment of responses. The analysis method reported here is the most fruitful of those we have considered. Since DATACOL is programmed in assembly language and RUNANAL is programmed in interpreter language (FOCAL 12), this approach also permits the implementation of different analysis strategies without requiring a major programming effort. Both programs are sufficiently general to be applicable to many problems in the field of neurobiology, but specific enough to be useful.

\section{DATA COLLECTION}

Three types of responses are collected by DATACOL; the individual evoked potential, the averaged evoked potential, and the unit poststimulus histogram over the number of samples averaged. Since the data analysis program employs the system tape indexes (DIAL), it is preferable to collect data in blocks of 18 where each block consists of eight individual gross potentials, the average of those eight, and the poststimulus

*Supported in part by Research Grant NS07661 (R.F.T.) from NINDS, Research Scientist Award MH06650 (R.F.T.), and Research Grant MH19314 from the NIMH (R.F.T.), Predoctoral Fellowship MH48912 (R.A.R.), and postdoctoral Fellowship MH35534 (T.J.T.) from the NIMH, Equipment Grant GB14665 from the National Science Foundation, and Research Training Grant MH11095 to the Department of Psychobiology, University of California at Irvine. histogram of the associated unit activity.

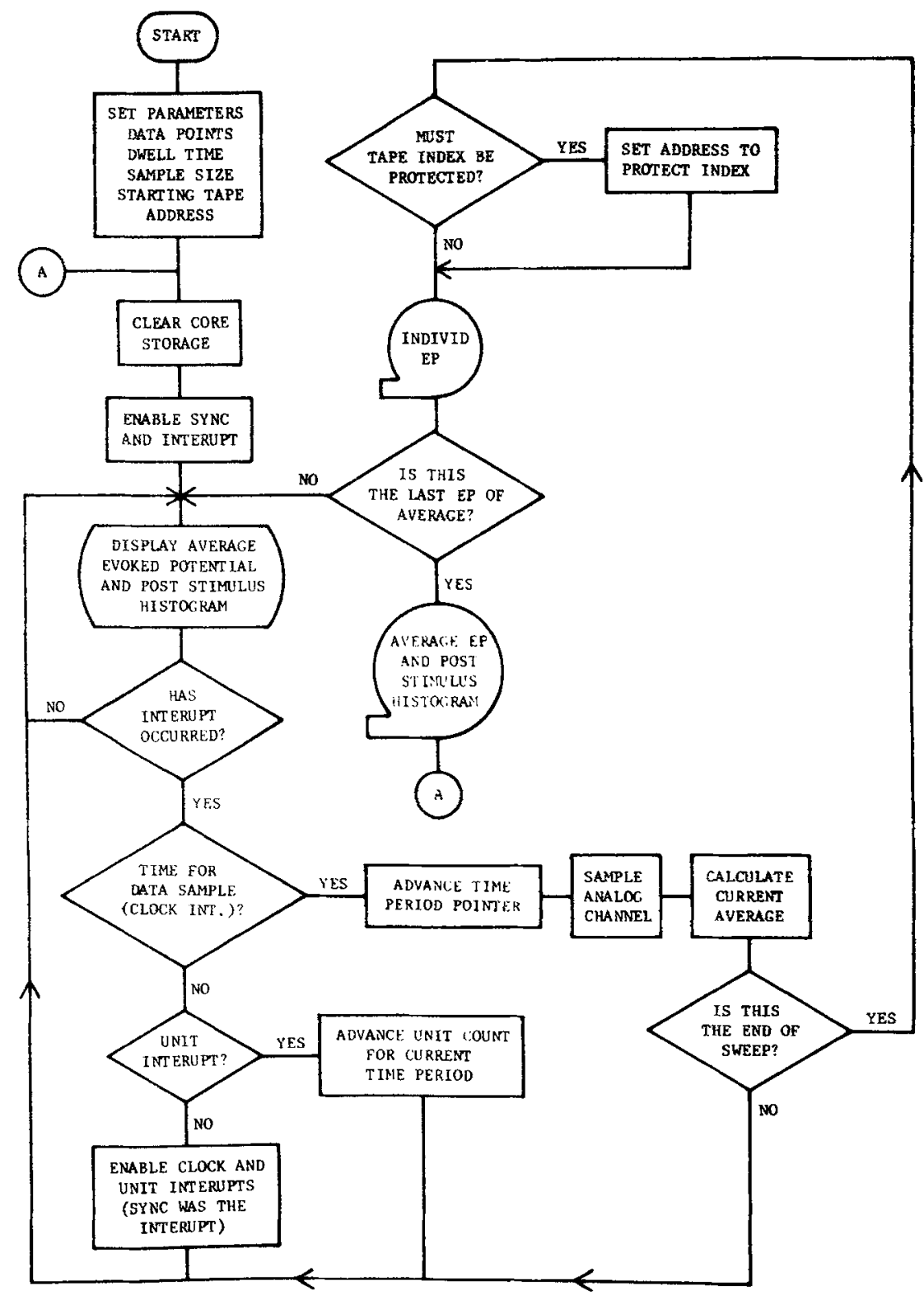

Fig. 1. Flow diagram for data collection (DATACOL).
The data collection program, DATACOL, permits the acquisition of data with the following parameters: number of data points per sweep, 1 to 256; dwell time per data point, 1 to $511 \mathrm{msec}$; number of samples per average, 1 to 512 in powers of 2 .

The tape address where the storage of data is to begin is also variable $(000$ 

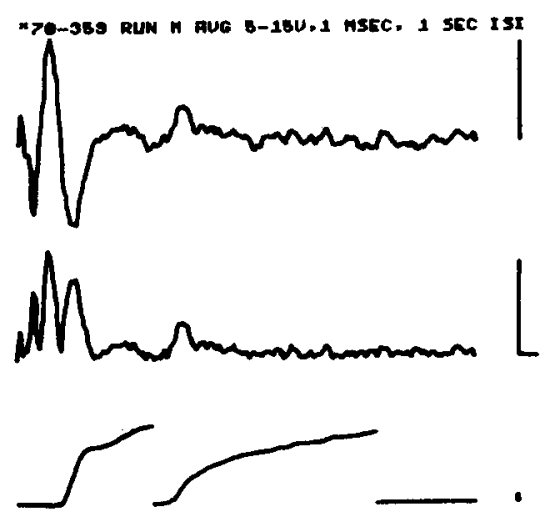

Fig. 2. Transformations of gross potential by RUNANEL. Top: Averaged wave form. Middle: Rectified wave form. Bottom: Integrations of rectified wave form from 25 to $75 \mathrm{msec}$ and from 75 to $200 \mathrm{msec}$.

to 777 on the PDP-12).

The flow logic for DATACOL is shown in Fig. 1.

\section{DATA ANALYSIS}

One method of analyzing evoked potentials has been to measure peak-to-peak voltage at various time points after stimulus presentation. The disadvantage with the approach is that much of the response is ignored, with the concurrent assumption that the majority of significant information is conveyed in the peak responses. During habituation paradigms in immobilized cat, activity less than $100 \mathrm{msec}$ poststimulus shows response habituation, while evoked activity from 100 to $250 \mathrm{msec}$ shows response sensitization (Teyler, Roemer, \& Thompson, 1971).

The analysis procedure described here is based upon full-wave rectification of the response. Rectification and integration of axonal tract potentials has been shown to be a valid procedure by Arduini and Pinneo (1962).

Rectification is accomplished by taking the absolute value of a wave form at successive time points. These time points are determined by the dwell time and number of bins selected in DATACOL. After rectification, the resultant is numerically integrated over successive, selectable time periods; the delta $t$ for the integration is, again, the dwell time from DATACOL. An example of this analysis procedure is shown in Fig. 2. The top wave form of Fig. 2 is an averaged gross evoked potential. The middle wave form is the result of rectification. The bottom wave form is the integral over two time periods, 25-75 and 75-200 msec.

The successive time periods of analysis are selectable. Consider a wave form with a time sweep of 250 msec.

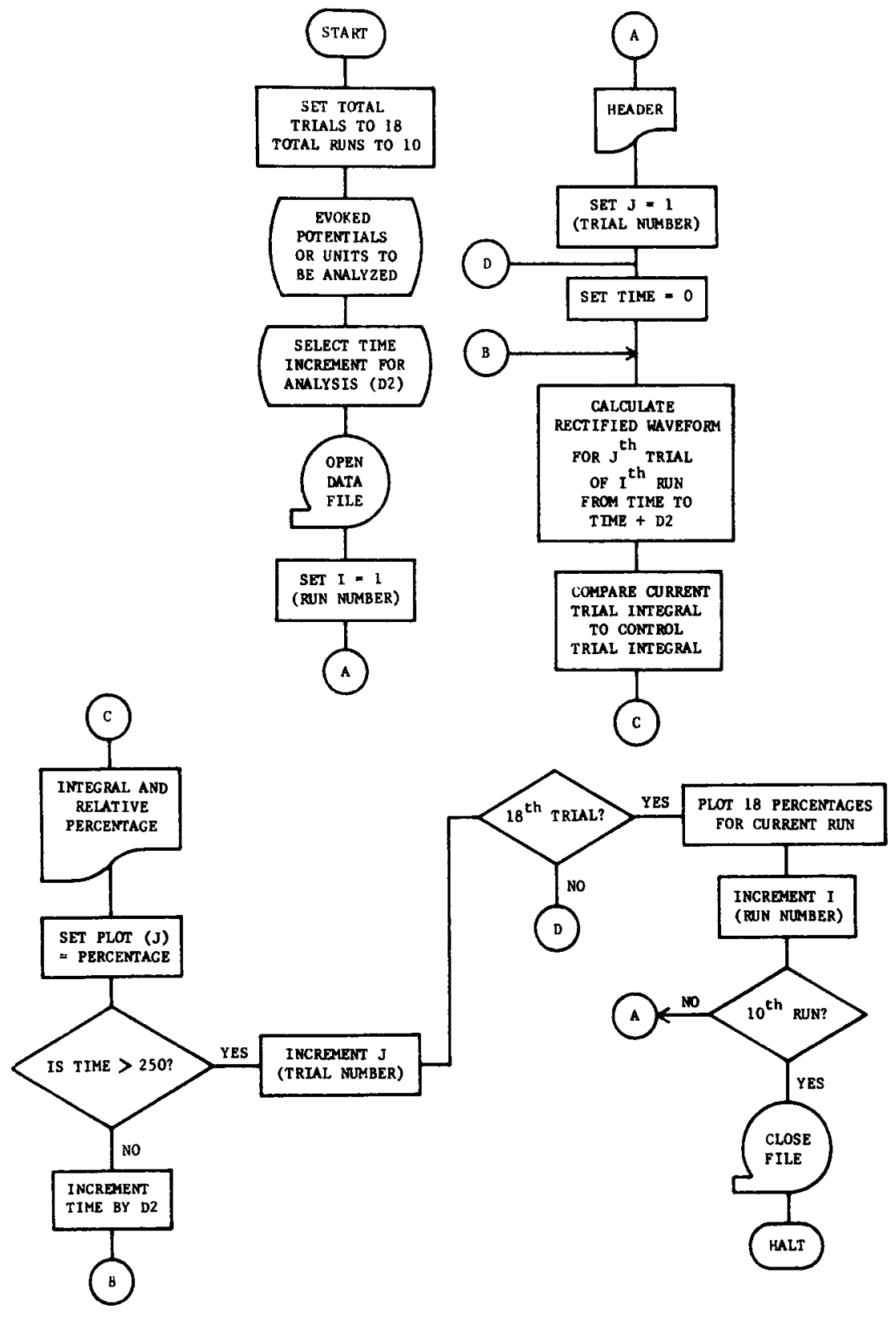

Fig. 3. Flow diagram for data analysis (RUNANAL).

RUNANAL can compare the control to the 17 test integrals in selectable time increments, e.g., $0-50,50-100$, 100-150, 150-200, and 200-250 msec. The smallest usable time increment is $1 \mathrm{msec}$ and the longest is the entire sweep length.

The analysis program, flow charted in Fig. 3, compares an integral obtained from a control or pretest situation with 17 subsequent experimental integrals over analagous time periods. The control integral is defined as $100 \%$; subsequent integrals are expressed in terms of percent of control. Therefore, percentages greater than 100 indicate an increase in activity over the control, while those less than $100 \%$ indicate a relative decrease in activity. 